\title{
SOME NEW PROPERTIES OF UNIVARIATE AND MULTIVARIATE GOTTLIEB POLYNOMIALS
}

\author{
ESRA ERKUŞ-DUMAN
}

Received 21 December, 2016

\begin{abstract}
The present study deals with some new properties for the Gottlieb polynomials in one and several variables. The results obtained here include various families of multilinear and multilateral generating functions, miscellaneous properties and also some special cases for these polynomials.
\end{abstract}

2010 Mathematics Subject Classification: 33C45

Keywords: Gottlieb polynomials, generating function, Pochhammer symbol, recurrence relation

\section{INTRODUCTION}

A theoretical connection with the unification of generating functions has great importance in the study of special functions. Steps forward in this directions has been made by some researchers $[1,2,7,8]$.

The (univariate) Gottlieb polynomials were introduced and investigated in 1938, and then have been cited in several articles. These polynomials are defined by [10]

$$
\begin{aligned}
\varphi_{n}(x ; \lambda) & =e^{-n \lambda} \sum_{k=0}^{n}\left(\begin{array}{l}
n \\
k
\end{array}\right)\left(\begin{array}{l}
x \\
k
\end{array}\right)\left(1-e^{\lambda}\right)^{k} \\
& =e^{-n \lambda}{ }_{2} F_{1}\left(-n,-x ; 1 ; 1-e^{\lambda}\right),
\end{aligned}
$$

where ${ }_{2} F_{1}$ denotes Gauss's hypergeometric series whose natural generalization of an arbitrary number of $p$ numerator and $q$ denominator parameters $\left(p, q \in \mathbb{N}_{0}:=\right.$ $\mathbb{N} \cup\{0\})$ is called and denoted by the generalized hypergeometric series ${ }_{p} F_{q}$ defined by

$$
\begin{aligned}
{ }_{p} F_{q}\left[\begin{array}{c}
\alpha_{1}, \ldots, \alpha_{p} ; \\
\beta_{1}, \ldots, \beta q ;
\end{array}\right] & =\sum_{n=0}^{\infty} \frac{\left(\alpha_{1}\right)_{n} \ldots\left(\alpha_{p}\right)_{n}}{\left(\beta_{1}\right)_{n} \ldots\left(\beta_{q}\right)_{n}} \frac{z^{n}}{n !} \\
& ={ }_{p} F_{q}\left(\alpha_{1}, \ldots, \alpha_{p} ; \beta_{1}, \ldots, \beta q ; z\right)
\end{aligned}
$$


Here $(\lambda)_{v}$ denotes the Pochhammer symbol defined (in terms of gamma function) by

$$
\begin{array}{rlr}
(\lambda)_{v} & =\frac{\Gamma(\lambda+v)}{\Gamma(\lambda)}\left(\lambda \in \mathbb{C} \backslash \mathbb{Z}_{0}^{-}\right) \\
& = \begin{cases}1, & \text { if } v=0 ; \lambda \in \mathbb{C} \backslash\{0\} \\
\lambda(\lambda+1) \ldots(\lambda+n-1), & \text { if } v=n \in \mathbb{N} ; \lambda \in \mathbb{C}\end{cases}
\end{array}
$$

and $\mathbb{Z}_{0}^{-}$denotes the set of nonpositive integers and $\Gamma(\lambda)$ is the familiar Gamma function. Furthermore, the hypergeometric function ${ }_{2} F_{1}$ has the following integral representation [12]:

$$
{ }_{2} F_{1}(a, b ; c ; z)=\frac{\Gamma(c)}{\Gamma(b) \Gamma(c-b)} \int_{0}^{1} t^{b-1}(1-t)^{c-b-1}(1-z t)^{-a} d t,
$$

where $\operatorname{Re}(c)>\operatorname{Re}(b)>0$, and $|\arg (1-z)|<\pi$.

Gottlieb [10] presented following generating functions for his polynomials $\varphi_{n}(x ; \lambda)$ (see also [12, p. 303]), which is denoted by $l_{n}(x)$ in [10],

$$
\begin{gathered}
\sum_{n=0}^{\infty} \varphi_{n}(x ; \lambda) t^{n}=(1-t)^{x}\left(1-t e^{-\lambda}\right)^{-x-1} \quad(|t|<1), \\
\sum_{n=0}^{\infty} \frac{(\mu)_{n}}{n !} \varphi_{n}(x ; \lambda) t^{n}=\left(1-t e^{-\lambda}\right)^{-\mu}{ }_{2} F_{1}\left[\begin{array}{c}
\mu,-x ; \\
1 ;
\end{array} \frac{\left(1-e^{-\lambda}\right) t}{1-t e^{-\lambda}}\right], \\
\sum_{n=0}^{\infty} \varphi_{n}(x ; \lambda) \frac{t^{n}}{n !}=e^{t}{ }_{1} F_{1}\left[x+1 ; 1 ;-\left(1-e^{-\lambda}\right) t\right] .
\end{gathered}
$$

In addition to this, we can easily see the following relation for the Gottlieb polynomials [14, p. 449, 20 (i)]:

$$
\sum_{n=0}^{\infty}\left(\begin{array}{c}
k+n \\
n
\end{array}\right) \varphi_{k+n}(x ; \lambda) t^{n}=(1-t)^{x-k}\left(1-t e^{-\lambda}\right)^{-x-1} \varphi_{k}\left(x ; \ln \left(\frac{e^{\lambda}-t}{1-t}\right)\right) .
$$

Recently, Khan and Akhlaq [11] introduced and investigated Gottlieb polynomials in two and three variables to give their generating functions. Choi [5], by modifying Khan and Akhlaq's method, showed how to generalize the Gottlieb polynomials in several variables to present two generating functions of the generalized Gottlieb polynomials. Furthermore, he derives $q$-extension of a generalization of Gottlieb polynomials in three variables and gives some formulas deducible from a generalization of these polynomials in several variables (see [4]). Choi introduced a several variable analogue of the Gottlieb polynomials as follows. 
Definition 1. An extension of the Gottlieb polynomials $\varphi_{n}(x ; \lambda)$ in $m$ variables is defined by [5]

$$
\begin{aligned}
& \varphi_{n}^{m}\left(x_{1}, \ldots, x_{m} ; \lambda_{1}, \ldots, \lambda_{m}\right)= \exp \left(-n \sigma_{m}\right) \sum_{r_{1}=0}^{n} \sum_{r_{2}=0}^{n-r_{1}} \sum_{r_{3}=0}^{n-r_{1}-r_{2}} \ldots \sum_{r_{m}=0}^{n-r_{1}-r_{2}-\ldots-r_{m-1}} \\
& \times \frac{(-n)_{\delta_{m}} \prod_{j=1}^{m}\left(-x_{j}\right)_{r_{j}} \prod_{j=1}\left(1-e^{\lambda_{j}}\right)^{r_{j}}}{\prod_{j=1}^{m} r_{j} ! \delta_{m} !}
\end{aligned}
$$

where $n, m \in \mathbb{N}$ and, for convenience,

$$
\sigma_{m}:=\sum_{j=1}^{m} \lambda_{j} \quad \text { and } \quad \delta_{m}:=\sum_{j=1}^{m} r_{j} .
$$

It is noted that the special case $m=1$ of (1.4) reduces immediately to the Gottlieb polynomials in (1.1). Multivariable Gottlieb polynomials defined by (1.4) have the following two generating functions [5]:

$$
\begin{aligned}
& \sum_{n=0}^{\infty} \varphi_{n}^{m}\left(x_{1}, \ldots, x_{m} ; \lambda_{1}, \ldots, \lambda_{m}\right) t^{n} \\
& =\left(1-t e^{-\sigma_{m}}\right)-\left(\sum_{j=1}^{m} x_{j}\right)-1 \prod_{j=1}^{m}\left(1-t e^{\lambda_{j}-\sigma_{m}}\right)^{x_{j}},
\end{aligned}
$$

where $m \in \mathbb{N}$ and $\sigma_{m}$ is given in (1.5).

$$
\begin{aligned}
& \sum_{n=0}^{\infty}(\mu)_{n} \varphi_{n}^{m}\left(x_{1}, \ldots, x_{m} ; \lambda_{1}, \ldots, \lambda_{m}\right) \frac{t^{n}}{n !} \\
= & \left(1-t e^{-\sigma_{m}}\right)^{-\mu} F_{D}^{(m)}\left[\mu,-x_{1}, \ldots,-x_{m} ; 1 ;\right. \\
& \left.\frac{t\left(e^{\lambda_{1}}-1\right) e^{-\sigma_{m}}}{1-t e^{-\sigma_{m}}}, \ldots, \frac{t\left(e^{\lambda_{m}}-1\right) e^{-\sigma_{m}}}{1-t e^{-\sigma_{m}}}\right],
\end{aligned}
$$

where $F_{D}^{(m)}$ [.] denotes one of the Lauricella series in $m$ variables [13, p.33, Eq.(4)] defined by

$$
\begin{aligned}
F_{D}^{(m)}\left[a, b_{1}, \ldots, b_{m} ; c ; x_{1}, \ldots, x_{m}\right] & =\sum_{r_{1}, r_{2}, \ldots, r_{m}=0}^{\infty} \frac{(a)_{\delta_{m}}\left(b_{1}\right)_{r_{1}} \ldots\left(b_{m}\right)_{r_{m}}}{(c)_{\delta_{m}}} \frac{x_{1}^{r_{1}}}{r_{1} !} \ldots \frac{x_{m}^{r_{m}}}{r_{m} !} \\
\left(\max \left\{\left|x_{1}\right|, \ldots,\left|x_{m}\right|\right\}<1\right) &
\end{aligned}
$$

and $\sigma_{m}, \delta_{m}$ are given in (1.5). 
The main object of this paper is to study different properties of Gottlieb polynomials in one and several variables. Various families of multilinear and multilateral generating functions are derived for these polynomials. Other miscellaneous properties of these multivariable polynomials are also discussed. Some special cases of the results presented in this study are also indicated.

\section{GENERATING FUNCTIONS}

In this section, firstly, we prove a theorem which gives bilateral generating functions relations for the Gottlieb polynomials defined by (1.1).

Theorem 1. Let

$$
N_{n, m, q}^{\mu, p}\left(y_{1}, \ldots, y_{s} ; z\right):=\sum_{k=0}^{[n / q]}\left(\begin{array}{c}
m+n \\
n-q k
\end{array}\right) a_{k} \Omega_{\mu+p k}\left(y_{1}, \ldots, y_{s}\right) z^{k} .
$$

If

$$
\Lambda_{m, q}\left[x ; \lambda ; y_{1}, \ldots, y_{s} ; t\right]=\sum_{n=0}^{\infty} a_{n} \varphi_{m+q n}(x ; \lambda) \Omega_{\mu+p n}\left(y_{1}, \ldots, y_{s}\right) t^{n},
$$

then, for every nonnegative integer $m$, we have

$$
\begin{aligned}
& \sum_{n=0}^{\infty} \varphi_{m+n}(x ; \lambda) N_{n, m, q}^{\mu, p}\left(y_{1}, \ldots, y_{s} ; z\right) t^{n} \\
= & (1-t)^{x-m}\left(1-t e^{-\lambda}\right)^{-x-1} \Lambda_{m, q}\left[x ; \ln \left(\frac{e^{\lambda}-t}{1-t}\right) ; y_{1}, \ldots, y_{s} ; z\left(\frac{t}{1-t}\right)^{q}\right] .
\end{aligned}
$$

Proof. If we denote the left-hand side of (2.2) by $T$ and use (2.1), then we obtain

$$
T=\sum_{n=0}^{\infty} \varphi_{m+n}(x ; \lambda) \sum_{k=0}^{[n / q]}\left(\begin{array}{c}
m+n \\
n-q k
\end{array}\right) a_{k} \Omega_{\mu+p k}\left(y_{1}, \ldots, y_{s}\right) z^{k} t^{n} .
$$

Replacing $n$ by $n+q k$ and then using relation (1.3) we may write

$$
\begin{aligned}
T= & \sum_{n=0}^{\infty} \sum_{k=0}^{\infty} \varphi_{m+n+q k}(x ; \lambda)\left(\begin{array}{c}
m+n+q k \\
n
\end{array}\right) a_{k} \Omega_{\mu+p k}\left(y_{1}, \ldots, y_{s}\right) z^{k} t^{n+q k} \\
= & \sum_{k=0}^{\infty}(1-t)^{x-m-q k}\left(1-t e^{-\lambda}\right)^{-x-1} \varphi_{m+q k}\left(x ; \ln \left(\frac{e^{\lambda}-t}{1-t}\right)\right) a_{k} \\
& \times \Omega_{\mu+p k}\left(y_{1}, \ldots, y_{s}\right)\left(z t^{q}\right)^{k} \\
= & (1-t)^{x-m}\left(1-t e^{-\lambda}\right)^{-x-1} \Lambda_{m, q}\left[x ; \ln \left(\frac{e^{\lambda}-t}{1-t}\right) ; y_{1}, \ldots, y_{s} ; z\left(\frac{t}{1-t}\right)^{q}\right],
\end{aligned}
$$

which completes the proof. 
Now we give a theorem which is about several families of bilinear and bilateral generating functions for the multivariable Gottlieb polynomials $\varphi_{n}^{m}\left(x_{1}, \ldots, x_{m} ; \lambda_{1}, \ldots, \lambda_{m}\right)$.

Theorem 2. Corresponding to an identically non-vanishing function $\Omega_{\mu}\left(y_{1}, \ldots, y_{s}\right)$ of $s$ complex variables $y_{1}, \ldots, y_{s}(s \in \mathbb{N})$ and of complex order $\mu$, let

$$
\Lambda_{\mu, \nu}\left(y_{1}, \ldots, y_{s} ; z\right):=\sum_{k=0}^{\infty} a_{k} \Omega_{\mu+\nu k}\left(y_{1}, \ldots, y_{s}\right) z^{k},
$$

where $a_{k} \neq 0, \mu, v \in \mathbb{C}$ and

$$
\begin{aligned}
& \Theta_{n, m, p}^{\mu, \nu}\left(x_{1}, \ldots, x_{m} ; \lambda_{1}, \ldots, \lambda_{m} ; y_{1}, \ldots, y_{s} ; \tau\right) \\
:= & \sum_{k=0}^{[n / p]} a_{k} \varphi_{n-p k}^{m}\left(x_{1}, \ldots, x_{m} ; \lambda_{1}, \ldots, \lambda_{m}\right) \Omega_{\mu+v k}\left(y_{1}, \ldots, y_{s}\right) \tau^{k}
\end{aligned}
$$

$(n, p \in \mathbb{N})$. Then we have

$$
\begin{aligned}
& \sum_{n=0}^{\infty} \Theta_{n, m, p}^{\mu, \nu}\left(x_{1}, \ldots, x_{m} ; \lambda_{1}, \ldots, \lambda_{m} ; y_{1}, \ldots, y_{s} ; \frac{\eta}{t^{p}}\right) t^{n} \\
& =\left(1-t e^{-\sigma_{m}}\right)^{-\left(\sum_{j=1}^{m} x_{j}\right)-1} \prod_{j=1}^{m}\left(1-t e^{\lambda_{j}-\sigma_{m}}\right)^{x_{j}} \Lambda_{\mu, \nu}\left(y_{1}, \ldots, y_{s} ; \eta\right)
\end{aligned}
$$

provided that each member of (2.4) exists.

Proof. If we denote the left-hand side of (2.4) by $S$ and use (2.3), then we obtain

$$
S=\sum_{n=0}^{\infty} \sum_{k=0}^{[n / p]} a_{k} \varphi_{n-p k}^{m}\left(x_{1}, \ldots, x_{m} ; \lambda_{1}, \ldots, \lambda_{m}\right) \Omega_{\mu+v k}\left(y_{1}, \ldots, y_{s}\right) \eta^{k} t^{n-p k} .
$$

Replacing $n$ by $n+p k$ we may write from the generating function (1.6) that

$$
\begin{aligned}
S & =\sum_{n=0}^{\infty} \sum_{k=0}^{\infty} a_{k} \varphi_{n}^{m}\left(x_{1}, \ldots, x_{m} ; \lambda_{1}, \ldots, \lambda_{m}\right) \Omega_{\mu+\nu k}\left(y_{1}, \ldots, y_{s}\right) \eta^{k} t^{n} \\
& =\sum_{n=0}^{\infty} \varphi_{n}^{m}\left(x_{1}, \ldots, x_{m} ; \lambda_{1}, \ldots, \lambda_{m}\right) t^{n} \sum_{k=0}^{\infty} a_{k} \Omega_{\mu+v k}\left(y_{1}, \ldots, y_{s}\right) \eta^{k} \\
& =\left(1-t e^{-\sigma_{m}}\right)-\left(\sum_{j=1}^{m} x_{j}\right)-1 \prod_{j=1}^{m}\left(1-t e^{\lambda_{j}-\sigma_{m}}\right)^{x_{j}} \Lambda_{\mu, \nu}\left(y_{1}, \ldots, y_{s} ; \eta\right),
\end{aligned}
$$

which completes the proof. 
It is possible to give many applications of our theorems with help of appropriate choices of the multivariable functions $\Omega_{\mu+\psi k}\left(y_{1}, \ldots, y_{s}\right), k \in \mathbb{N}_{0}, s \in \mathbb{N}$.

For example, set $s=m$ and take $\Omega_{\mu+v k}\left(y_{1}, \ldots, y_{m}\right)=h_{\mu+v k}^{\left(\beta_{1}, \ldots, \beta_{m}\right)}\left(y_{1}, \ldots, y_{m}\right)$ in Theorem 2. Recall that, by $h_{n}^{\left(\alpha_{1}, \ldots, \alpha_{r}\right)}\left(x_{1}, \ldots, x_{r}\right)$, we denote the multivariable Lagrange-Hermite polynomials (see, e.g., [2]) generated by

$$
\prod_{j=1}^{r}\left\{\left(1-x_{j} t^{j}\right)^{-\alpha_{j}}\right\}=\sum_{n=0}^{\infty} h_{n}^{\left(\alpha_{1}, \ldots, \alpha_{r}\right)}\left(x_{1}, \ldots, x_{r}\right) t^{n},
$$

where $|t|<\min \left\{\left|x_{1}\right|^{-1},\left|x_{2}\right|^{-1 / 2}, \ldots,\left|x_{r}\right|^{-1 / r}\right\}$. We also know that

$$
h_{n}^{\left(\alpha_{1}, \ldots, \alpha_{r}\right)}\left(x_{1}, \ldots, x_{r}\right)=\sum_{k_{1}+2 k_{2}+\ldots+r k_{r}=n}\left(\alpha_{1}\right)_{k_{1}} \ldots\left(\alpha_{r}\right)_{k_{r}} \frac{x_{1}^{k_{1}}}{k_{1} !} \ldots \frac{x_{r}^{k_{r}}}{k_{r} !} .
$$

Then, from Theorem 2, we obtain the following result which is a class of bilateral generating functions for the multivariable Lagrange-Hermite polynomials and the multivariable Gottlieb polynomials.

Corollary 1. If

$$
\Lambda_{\mu, v}\left(y_{1}, \ldots, y_{m} ; z\right):=\sum_{k=0}^{\infty} a_{k} h_{\mu+v k}^{\left(\beta_{1}, \ldots, \beta_{m}\right)}\left(y_{1}, \ldots, y_{m}\right) z^{k}, \quad\left(a_{k} \neq 0, \mu, v \in \mathbb{C}\right),
$$

and

$$
\begin{aligned}
& \Theta_{n, m, p}^{\mu, \nu}\left(x_{1}, \ldots, x_{m} ; \lambda_{1}, \ldots, \lambda_{m} ; y_{1}, \ldots, y_{m} ; \tau\right) \\
:= & \sum_{k=0}^{[n / p]} a_{k} \varphi_{n-p k}^{m}\left(x_{1}, \ldots, x_{m} ; \lambda_{1}, \ldots, \lambda_{m}\right) h_{\mu+\nu k}^{\left(\beta_{1}, \ldots, \beta_{m}\right)}\left(y_{1}, \ldots, y_{m}\right) \tau^{k},
\end{aligned}
$$

where $n, p \in \mathbb{N}$, then we have

$$
\begin{aligned}
& \sum_{n=0}^{\infty} \Theta_{n, m, p}^{\mu, v}\left(x_{1}, \ldots, x_{m} ; \lambda_{1}, \ldots, \lambda_{m} ; y_{1}, \ldots, y_{m} ; \frac{\eta}{t^{p}}\right) t^{n} \\
& =\left(1-t e^{-\sigma_{m}}\right)-\left(\sum_{j=1}^{m} x_{j}\right)-1 \prod_{j=1}^{m}\left(1-t e^{\lambda_{j}-\sigma_{m}}\right)^{x_{j}} \Lambda_{\mu, \nu}\left(y_{1}, \ldots, y_{m} ; \eta\right)
\end{aligned}
$$

provided that each member of (2.6) exists.

Remark 1. Using the generating relation (2.5) for the multivariable LagrangeHermite polynomials and taking $a_{k}=1, \mu=0, v=1$, we have

$$
\sum_{n=0}^{\infty} \sum_{k=0}^{[n / p]} \varphi_{n-p k}^{m}\left(x_{1}, \ldots, x_{m} ; \lambda_{1}, \ldots, \lambda_{m}\right) h_{k}^{\left(\beta_{1}, \ldots, \beta_{m}\right)}\left(y_{1}, \ldots, y_{m}\right) \eta^{k} t^{n-p k}
$$




$$
=\left(1-t e^{-\sigma_{m}}\right)-\left(\sum_{j=1}^{m} x_{j}\right)-1 \prod_{j=1}^{m}\left(1-t e^{\lambda_{j}-\sigma_{m}}\right)^{x_{j}}\left(1-y_{j} \eta^{j}\right)^{-\beta_{j}},
$$

where $\sigma_{m}:=\sum_{j=1}^{m} \lambda_{j}$ and $|\eta|<\min \left\{\left|y_{1}\right|^{-1},\left|y_{2}\right|^{-1 / 2}, \ldots,\left|y_{m}\right|^{-1 / m}\right\}$.

Also, choosing $s=m$ and $\Omega_{\mu+v k}\left(y_{1}, \ldots, y_{m}\right)=\varphi_{\mu+\nu k}^{m}\left(y_{1}, \ldots, y_{m} ; \lambda_{1}, \ldots, \lambda_{m}\right)$, $\mu, v \in \mathbb{N}_{0}$, in Theorem 2, we obtain the following class of bilinear generating functions for the multivariable Gottlieb polynomials defined by (1.4).

Corollary 2. If

$\Lambda_{\mu, v}\left(y_{1}, \ldots, y_{m} ; z\right):=\sum_{k=0}^{\infty} a_{k} \varphi_{\mu+v k}^{m}\left(y_{1}, \ldots, y_{m} ; \lambda_{1}, \ldots, \lambda_{m}\right) z^{k}, \quad\left(a_{k} \neq 0, \mu, v \in \mathbb{C}\right)$,

and

$$
\begin{aligned}
& \Theta_{n, m, p}^{\mu, \nu}\left(x_{1}, \ldots, x_{m} ; \lambda_{1}, \ldots, \lambda_{m} ; y_{1}, \ldots, y_{m} ; \tau\right) \\
:= & \sum_{k=0}^{[n / p]} a_{k} \varphi_{n-p k}^{m}\left(x_{1}, \ldots, x_{m} ; \lambda_{1}, \ldots, \lambda_{m}\right) \varphi_{\mu+v k}^{m}\left(y_{1}, \ldots, y_{m} ; \lambda_{1}, \ldots, \lambda_{m}\right) \tau^{k},
\end{aligned}
$$

where $n, p \in \mathbb{N}$, then we have

$$
\begin{aligned}
& \sum_{n=0}^{\infty} \Theta_{n, m, p}^{\mu, \nu}\left(x_{1}, \ldots, x_{m} ; \lambda_{1}, \ldots, \lambda_{m} ; y_{1}, \ldots, y_{m} ; \frac{\eta}{t^{p}}\right) t^{n} \\
& =\left(1-t e^{-\sigma_{m}}\right)-\left(\sum_{j=1}^{m} x_{j}\right)-1 \prod_{j=1}^{m}\left(1-t e^{\lambda_{j}-\sigma_{m}}\right)^{x_{j}} \Lambda_{\mu, \nu}\left(y_{1}, \ldots, y_{m} ; \eta\right)
\end{aligned}
$$

provided that each member of (2.7) exists.

Remark 2. Using the generating relation (1.6) for the multivariable Gottlieb polynomials and taking $a_{k}=1, \mu=0, v=1$, we have

$$
\begin{aligned}
& \sum_{n=0}^{\infty} \sum_{k=0}^{[n / p]} \varphi_{n-p k}^{m}\left(x_{1}, \ldots, x_{m} ; \lambda_{1}, \ldots, \lambda_{m}\right) \varphi_{k}^{m}\left(y_{1}, \ldots, y_{m} ; \lambda_{1}, \ldots, \lambda_{m}\right) \eta^{k} t^{n-p k} \\
&=\left(1-t e^{-\sigma_{m}}\right)-\left(\sum_{j=1}^{m}\left(x_{j}+y_{j}\right)\right)-2 \\
& \prod_{j=1}^{m}\left(1-t e^{\lambda_{j}-\sigma_{m}}\right)^{x_{j}}\left(1-\eta e^{\lambda_{j}-\sigma_{m}}\right)^{y_{j}},
\end{aligned}
$$

where $\sigma_{m}:=\sum_{j=1}^{m} \lambda_{j}$ 
Furthermore, for every suitable choice of the coefficients $a_{k}\left(k \in \mathbb{N}_{0}\right)$, if the multivariable function $\Omega_{\mu+\psi k}\left(y_{1}, \ldots, y_{s}\right)(s \in \mathbb{N})$ is expressed as an appropriate product of several simpler functions, the assertions of Theorems 1 and 2 can be applied in order to derive various families of multilinear and multilateral generating functions for the univariate and multivariate Gottlieb polynomials.

\section{Miscellaneous PROPERTies}

In this section, firstly, we obtain a recurrence relation for the multivariable Gottlieb polynomials. Then, using the relation between Gottlieb and Jacobi polynomials, we derive some properties for the Gottlieb polynomials.

Theorem 3. Multivariable Gottlieb polynomials have the following recurrence relation:

$$
\begin{gathered}
(n+1) \varphi_{n+1}^{m}+e^{\lambda_{i}-2 \sigma_{m}} n \varphi_{n-1}^{m}=e^{-\sigma_{m}}\left(1+n+n e^{\lambda_{i}}+\sum_{j=1}^{m} x_{j}\left(1-e^{\lambda_{j}}\right)\right) \varphi_{n}^{m}, \\
(i=1, \ldots, m ; n \geq 2) .
\end{gathered}
$$

Proof. By differentiating each member of the generating function relation (1.6) with respect to $t$ and making some necessary adjustments and then identifying the corresponding coefficients of $t^{n}$, we arrive at the desired recurrence relation.

It is known that

$$
\varphi_{n}(x ; \lambda)=P_{n}^{(0, x-n)}\left(2 e^{-\lambda}-1\right),
$$

where the classical Jacobi polynomials $P_{n}^{(\alpha, \beta)}(z)$ are defined by [12]

$$
P_{n}^{(\alpha, \beta)}(z)=\sum_{k=0}^{n}\left(\begin{array}{c}
n+\alpha \\
k
\end{array}\right)\left(\begin{array}{c}
n+\beta \\
n-k
\end{array}\right)\left(\frac{x+1}{2}\right)^{k}\left(\frac{x-1}{2}\right)^{n-k} .
$$

Equation (3.1) can be used to deduce numerous properties and characteristics of the Gottlieb polynomials from those of the Jacobi polynomials.

In 1966, Izuru Fujiwara studied extended Jacobi polynomials $F_{n}^{(\alpha, \beta)}(x ; a, b, c)$ which are defined by the Rodrigues formula [9]

$$
\begin{aligned}
F_{n}^{(\alpha, \beta)}(x ; a, b, c)= & \frac{(-c)^{n}}{n !}(x-a)^{-\alpha}(b-x)^{-\beta} \\
& \times \frac{d^{n}}{d x^{n}}\left\{(x-a)^{n+\alpha}(b-x)^{n+\beta}\right\} \quad(c>0) .
\end{aligned}
$$

Szegö [15] showed that $F_{n}^{(\alpha, \beta)}(x ; a, b, c)$ polynomials are a constant multiple of the classical Jacobi polynomials $P_{n}^{(\alpha, \beta)}(x)$ in the form

$$
F_{n}^{(\alpha, \beta)}(x ; a, b, c)=\{c(a-b)\}^{n} P_{n}^{(\alpha, \beta)}\left(\frac{2(x-a)}{a-b}+1\right)
$$


or, equivalently,

$$
P_{n}^{(\alpha, \beta)}(x)=\{c(a-b)\}^{-n} F_{n}^{(\alpha, \beta)}\left(\frac{1}{2}\{a+b+(a-b) x\} ; a, b, c\right) .
$$

In addition to this, we know that the following equality [3]:

$$
g_{n}^{(\alpha, \beta)}(x, y)=(y-x)^{n} P_{n}^{(-\alpha-n,-\beta-n)}\left(\frac{x+y}{x-y}\right),
$$

where $g_{n}^{(\alpha, \beta)}(x, y)$ are known as the Lagrange polynomials generated by [6]

$$
\begin{aligned}
(1-x t)^{-\alpha}(1-y t)^{-\beta}= & \sum_{n=0}^{\infty} g_{n}^{(\alpha, \beta)}(x, y) t^{n} \\
& \left(|t|<\min \left\{|x|^{-1},|y|^{-1}\right\}\right) .
\end{aligned}
$$

Theorem 4. For the Gottlieb polynomials $\varphi_{n}(x ; \lambda)$, we have

(i) $\varphi_{n}(x ; \lambda)=\{c(a-b)\}^{-n} F_{n}^{(0, x-n)}\left(\frac{1}{2}\left\{a+b+(a-b)\left(2 e^{-\lambda}-1\right)\right\} ; a, b, c\right)$

(ii) $\varphi_{n}\left(x ; \ln \frac{a-b}{a}\right)=(b-a)^{-n} g_{n}^{(-n,-x)}(a, b)$.

Proof. (i) Using relations (3.1) and (3.2), we arrived the result.

(ii) Using relations (3.1) and (3.3), we get the desired result.

Theorem 5. Gottlieb polynomials have the following integral representation:

$$
\varphi_{n}(x ; \lambda)=-\frac{\sin \pi x}{\pi e^{n \lambda}} \int_{0}^{1} t^{-x-1}(1-t)^{x}\left(1-\left(1-e^{\lambda}\right) t\right)^{n} d t,
$$

where $-1<x<0$.

Proof. If we use definition of Gottlieb polynomials given by (1.1) in (1.2) and make some necessary calculation, we easily arrived this result.

Finally, using the fact that (see [14])

$$
P_{n}^{(\alpha, \beta)}(x)=\frac{1}{\Gamma(\alpha+\beta+n+1)} \int_{0}^{\infty} t^{\alpha+\beta+n} e^{-t} L_{n}^{(\alpha)}\left(\frac{(1-x) t}{2}\right) d t,
$$

where $L_{n}^{(\alpha)}(x)$ are the Laguerre polynomials given by the Rodrigues formula [12]

$$
L_{n}^{(\alpha)}(x)=\frac{x^{-\alpha} e^{x}}{n !} \frac{d^{n}}{d x^{n}}\left(e^{-x} x^{n+\alpha}\right),
$$

and also considering (3.1), we immediately obtain the following result. 
Theorem 6. Gottlieb polynomials have the following integral representation:

$$
\varphi_{n}(x ; \lambda)=\frac{1}{x \Gamma(x)} \int_{0}^{\infty} t^{x} e^{-t} L_{n}\left(\left(1-e^{-\lambda}\right) t\right) d t .
$$

\section{ACKNOWLEDGEMENT}

The author would like to thank the referees for carefully reading the manuscript.

\section{REFERENCES}

[1] A. Altın, R. Aktaş, and E. Erkuş-Duman, "On a multivariable extension for the extended Jacobi polynomials.” J. Math. Anal. Appl., vol. 353, no. 1, pp. 121-133, 2009, doi: 10.1016/j.jmaa.2008.11.070.

[2] A. Altın and E. Erkuş, "On a multivariable extension of the Lagrange-Hermite polynomials." Integral Transform. Spec. Funct., vol. 17, no. 4, pp. 239-244, 2006, doi: 10.1080/10652460500432006.

[3] W.-C. C. Chan, C.-J. Chyan, and H. M. Srivastava, "The Lagrange polynomials in several variables." Integral Transform. Spec. Funct., vol. 12, no. 2, pp. 139-148, 2001, doi: 10.1080/10652460108819340.

[4] J. Choi and H. M. Srivastava, "q-extension of a multivariable and multiparameter generalization of the gottlieb polynomials in several variables.” Tokyo J. Math., vol. 37, no. 1, pp. 111-125, 2014, doi: $10.3836 / \mathrm{tjm} / 1406552433$.

[5] J. Choi, "A generalization of Gottlieb polynomials in several variables." Appl. Math. Lett., vol. 25, no. 1, pp. 43-46, 2012, doi: 10.1016/j.aml.2011.07.006.

[6] A. Erdélyi, W. Magnus, F. Oberhettinger, and F. G. Tricomi, Higher transcendental functions. Vol. III. McGraw-Hill Book Company, Inc., New York-Toronto-London, 1955.

[7] E. Erkuş and H. M. Srivastava, "A unified presentation of some families of multivariable polynomials." Integral Transforms Spec. Funct., vol. 17, no. 4, pp. 267-273, 2006, doi: 10.1080/10652460500444928.

[8] E. Erkuş-Duman, A. Altın, and R. Aktaş, "Miscellaneous properties of some multivariable polynomials." Math. Comput. Modelling., vol. 54, no. 9-10, pp. 1875-1885, 2011, doi: 10.1016/j.mcm.2011.04.010.

[9] I. Fujiwara, "A unified presentation of classical orthogonal polynomials." Math. Japon., vol. 11, pp. 133-148, 1966.

[10] M. J. Gottlieb, "Concerning some polynomials orthogonal on a finite or enumerable set of points." Amer. J. Math., vol. 60, no. 2, pp. 453-458, 1938, doi: 10.2307/2371307.

[11] M. A. Khan and M. Akhlaq, "Some new generating functions for gottlieb polynomials of several variables.” Int. Trans. Appl. Sci., vol. 1, no. 4, pp. 567-570, 2009.

[12] E. D. Rainville, Special functions. The Macmillan Co., New York, 1960.

[13] H. M. Srivastava and P. W. Karlsson, Multiple Gaussian hypergeometric series., ser. Ellis Horwood Series: Mathematics and its Applications. Ellis Horwood Ltd., Chichester; Halsted Press [John Wiley \& Sons, Inc.], New York, 1985.

[14] H. M. Srivastava and H. L. Manocha, A treatise on generating functions., ser. Ellis Horwood Series: Mathematics and its Applications. Ellis Horwood Ltd., Chichester; Halsted Press [John Wiley \& Sons, Inc.], New York, 1984.

[15] G. Szegô, Orthogonal polynomials., 4th ed. American Mathematical Society, Providence, R.I., 1975, american Mathematical Society, Colloquium Publications, Vol. XXIII. 
Author's address

\section{Esra Erkuş-Duman}

Gazi University, Department of Mathematics, Teknikokullar TR-06500, Ankara, Turkey

E-mail address: eduman@gazi.edu.tr; eerkusduman@gmail.com 